\title{
Research On Intelligent Low Carbon Urban Lighting System
}

\section{Tao Wang ${ }^{1, \text { a }}$, Zhanhua Zan ${ }^{2, b}$,Zhenlong Yang ${ }^{4, d}$,Jinlong Zhu ${ }^{5, f}$, Longfei Ma ${ }^{6, \mathrm{~g}}$, Yapeng $\mathrm{Hu}^{7, \mathrm{~h}}$, Xinchao Zhao ${ }^{8, \mathrm{i}}$}

${ }^{1}$ Academy of mechanical engineering of Hebei University of Technology, Tianjin 300230,China.

${ }^{2}$ Academy of mechanical engineering of Hebei University of Technology, Tianjin 300230, China.

${ }^{3}$ Wuxi Jin Tianyang laser Electronics Co. Ltd, Wuxi,Jiangsu 213692,China.

awtao_1@163.com , banhua13@sina.com,

\begin{abstract}
Keywords: Intelligent low carbon lighting system Lighting System Data Fusion Fuzzy Control Neural Network

Abstract. Smart low-carbon city as a rapidly developing field is the most investment value of the new project in the 21st century.In this paper, we propose an intelligent low carbon urban lighting system using fuzzy control and neural network control algorithm, it can realize intelligent, low carbonization and human nature of urban .
\end{abstract}

\section{Intelligent low-carbon urban lighting system overview}

Intelligent low-carbon city is in the background of the urban facilities, buildings, roads, community , the integration of advanced network communication technology, automatic control technology, embedded technology and so on ,achieving "the wisdom of low-carbon city.”Application of fuzzy control and neural network control algorithm, this paper puts forward a intelligent low carbon city lighting system, introduction of multi-sensor data fusion technology, according to characteristics of intelligent low carbon city road, the fuzzy control and neural network control technology embedded into the control algorithm of lighting system, the overall framework of the lighting control system was constructed,using CC2530, STM32 as control chip, using the IEEE. 802.15 as the software design,according to $\mathrm{Z}$ - stack protocol stack wireless communication program design, to realize the communication between wireless lighting nodes and the main controller,by adjusting the fuzzy rules and membership functions of the fuzzy control system ,the BP neural network control mode based on Mamdani is determined, which can realize the intelligent, low carbon and human nature of the intelligent low-carbon city, meet the needs of low-carbon city, improve the quality of life, reduce operating costs and achieve energy saving and environmental protection.

\section{System design.}

Design principles of smart low-carbon city: lower cost, energy saving, simple and practical, scalable. Based on intelligent low carbon urban lighting system of STM32 and 2.4G wireless control is mainly composed of intelligent low carbon city control center, wireless lighting control node, network interface and lighting control module ${ }^{[1]}$,the overall schematic diagram of the lighting system is shown in Figure 2.1.

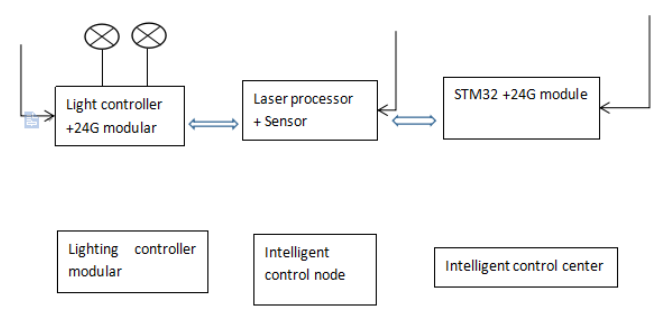

Figure 2.1 The overall schematic diagram of the lighting system 


\section{Research on the algorithm of intelligent low carbon urban lighting control system}

Structure model of BP neural network algorithm . BP(Back Propagation) neural network is a kind of neural network with deviation back propagation function. It is also a kind of one-way transmission of multi level network ${ }^{[2]}$, BP neural network structure is shown in Figure 3.1.

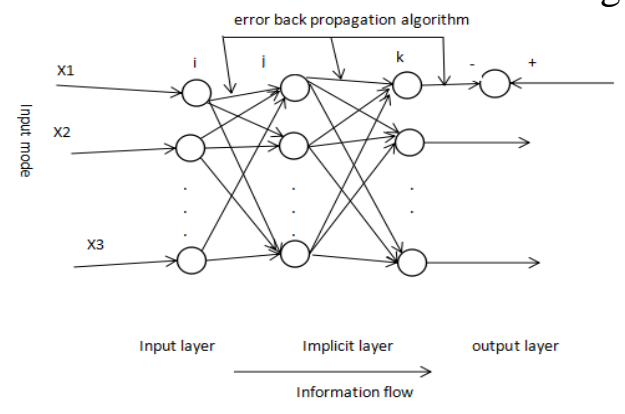

Figure 3.1 Structure of BP neural network

BP neural network has an input layer, a hidden layer and output layer,hidden layer only with the input layer and the output layer has a close relationship, no contact with the outside world, the network will correspond to a n-dimensional input, m-dimensional output node, it can be concluded that it is a nonlinear mapping from the n-dimensional input space to m dimensional output space.

Analysis of the learning formula of BP neural network algorithm

$$
\text { net }_{j}=\sum_{i=1}^{M} w_{i j} O_{i}
$$

1)Correction formula for weight coeffient of any neuron:

$$
\square w_{j k}=\eta\left(\mathrm{d}_{k}-O_{k}\right) f^{\prime}\left(\text { net }_{k}\right)\left(\bigotimes_{j} 1 \neq \eta \delta_{k} O_{j}=\eta O_{k}\left(1-O_{k}\right)\left(\mathrm{d}_{k}-O_{k}\right) \mathrm{O}_{j}\right.
$$

2)Adjustment of node weight coefficient of implicit layer

The change of weight coefficient:

$$
\begin{gathered}
-\frac{\partial E}{\partial O_{j}}=-\sum_{k=1}^{L} \frac{\partial E}{\partial n e t_{k}} \frac{\partial n e t_{j k}}{\partial O_{j}}=\sum_{k=1}^{L}\left(-\frac{\partial E}{\partial n e t_{k}}\right) \frac{\partial}{\partial O_{j}}\left(\sum_{j=1}^{q} w_{j k} O_{j()}\right) \\
=\sum_{k=1}^{L}\left(-\frac{\partial E}{\partial n e t_{k}}\right) w_{j k}^{(3-2)}=\sum_{k=1}^{L} \delta_{k} w_{j k} \\
\delta_{j} \bar{T} f_{3}^{\prime}\left(\mathrm{net}_{j}\right) \sum_{k=1}^{L} \delta_{k} w_{j k}
\end{gathered}
$$

From the above formula:

$$
\square w_{i j}=\eta f^{\prime}\left(\text { net }_{j}\right)\left(\sum^{L} \delta_{k} w_{j k}\right)\left(3_{i} \text { 4) } \eta O_{j}\left(1-\mathrm{O}_{j}\right)\left(\sum^{L} \delta_{k} \mathrm{w}_{j k}\right) \mathrm{O}_{i}\right.
$$

From the results above, the weights of the network can be adjutsted.:

$$
w_{i j}(\mathrm{t}+1)=\mathrm{w}_{i j}(\mathrm{t})+\eta \delta_{i} O_{i}+\alpha\left[\mathrm{w}_{i j}(\mathrm{t})-\mathrm{w}_{i j}(\mathrm{t}-1)\right]
$$

In the formula, $\mathrm{t}+1$ represents step $\mathrm{t}+1$, as the smoothing factor, $0<\alpha<1$ 。

Fuzzy reasoning algorithm. Mamdani reasoning: Using Mamdani fuzzy reasoning method, The fuzzy inference rules of multiple input single output Mamdani are as follows:

$$
R_{i}: \text { if } x_{1} \text { is } A_{1}^{i} \text { and } x_{2} \text { and } \cdots \text { and } x_{p} \text { is } A_{p}^{i} \text { then } y^{i} \text { is } B^{i} \text { (3-6) }
$$

Thereinto, $\mathrm{i}=1,2, \cdots, \mathrm{N}$ represents $\mathrm{N}$ fuzzy rules, ${ }^{x_{j}}$ as input variables, ${ }^{X}$ as the domain space ; $A_{j}^{i}$ as the fuzzy space of the antecedent variables; y as output of the fuzzy inference system, Y as the
domaion space; $B^{i}$ as the fuzzy domain of output variable. 
Takagi-Sugeno reasoning: Regarding the system's variables and input variables as the fuzzy control rules, using IF-THEN function to carry out reasoning ${ }^{[3]}$. The fuzzy control rules of T-S reasoning are as follows:

$$
R_{i}:_{\text {if }} x_{1} \text { is } A_{1}^{i} \text { and } x_{2} \text { and } \cdots \text { and } x_{p} \text { is } A_{p}^{i} \text { then } y^{i} \text { is } y^{i}=f_{i}\left(\mathrm{x}_{1}, \mathrm{x}_{2}, \ldots, \mathrm{x}_{p}\right)
$$

Thereinto ,i=1, 2, $\cdots, \mathrm{N}$ represents $\mathrm{N}$ fuzzy rules, ${ }^{X_{j}}$ as input variables, ${ }^{X}{ }_{j}$ as the domain space; $\square^{i}$

$A_{j}$ as the fuzzy space of the antecedent variables; $\mathrm{y}$ as output of the fuzzy inference system, $\mathrm{Y}$ as the domaion space; $f_{i}\left(\mathrm{x}_{1}, \mathrm{x}_{2}, \ldots, \mathrm{x}_{p}\right)$ as a linear or nonlinear function of the variable $\mathrm{x}$.

Precision design: Adopting the following methods: 1)Weighted average method, 2)gravity model approach, 3)Maximum membership function method, the maximum membership function method does not take into account the shape of the membership function, and only cares about the exact value of the maximum membership function of fuzzy space.

Low-carbon city of intelligent lighting system model of comprehensive fuzzy control and neural network. Based on BP neural network algorithm of Mamdani inference mode,Structure diagram is shown in Figure 3.2,

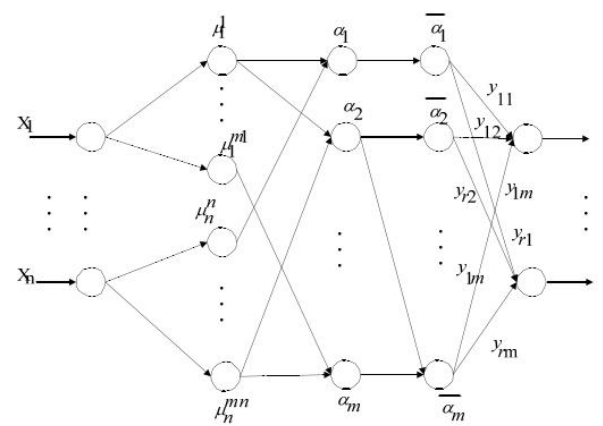

Figure 3.2 structure diagram of fuzzy neural network based on Mamdani reasoning

This is a five layer network structure, the specific structure of each layer is described as follows:

The first layer is the input layer, the input vector of the input layer ${ }_{i}$ is directly connected with each node of the first layer, so that the input vector is passed to the next layer.

The second layer is fuzzy language variables, such as PS, ZE, NS, etc, $\mu_{i}^{j}=\mu_{A_{i}^{j}}\left(x_{i}\right)$ $i=1,2, \ldots, n, j=1,2, \ldots, m, A_{i}^{j}$ 。 It makes the input of the upper level assigned to single fuzzy space,to obtain the membership function, $\mu_{i}^{j}=\mu_{A_{i}^{j}}\left(\mathrm{x}_{i}\right) i=1,2, \ldots, n, j=1,2, \ldots, m, A_{i}^{j}$ represents a fuzzy space.

The third layer is a fuzzy rule control layer, each node represents a fuzzy control rule , is to evaluate the feasibility of each rule.

The fourth layer is to realize the normalization process, so it must be the same as the number of nodes of the upper layer.

The fifth layer is the defuzzification layer,and the output layer, In order to get the exact output value , $i=1,23, \ldots . r$.

The algorithm is as follows:

Implicit layer(Two,three, four,):

Output layer:

$$
X^{(1)}(\mathrm{k})=\mathrm{f}\left(\mathrm{y}^{(1)}(\mathrm{k})\right)
$$

Weight of output layer:

$$
d_{j}^{(2)}(\mathrm{k})=\mathrm{f}^{-1}(\mathrm{o},(\mathrm{k}))
$$

Thereinto, Calman gain g:

$$
W_{j}^{(2)}(\mathrm{k})=\mathrm{W}_{j}^{(2)}(\mathrm{k}-1)+\mathrm{g}^{(1)}(\mathrm{k})\left[\mathrm{d}_{j}^{(2)}(\mathrm{k})-\mathrm{X}^{(1) \mathrm{T}}(\mathrm{k}) \mathrm{W}_{j}^{(2)}(\mathrm{k}-1)\right]
$$

$$
g^{(2)}(\mathrm{k})=\frac{p^{(2)}(\mathrm{k}-1) \mathrm{X}^{(1)}(\mathrm{k})}{\lambda+X^{(1) \mathrm{T}} \mathrm{p}^{(2)}(\mathrm{k}-1) \mathrm{X}^{(1)}(\mathrm{k})}
$$


Inverse correlation matrix p:

$$
\begin{aligned}
& p^{(1)}(\mathrm{k})=\frac{p^{(1)}(\mathrm{k}-1)}{\lambda+f\left(\mathrm{y}_{j}^{(1)}(\mathrm{k}) \mathrm{X}^{T}(\mathrm{k}) \mathrm{p}^{(1)} \mathrm{X}(\mathrm{k})\right)} \\
& \text { Output deviation formula: } \quad \varepsilon_{j}^{(2)}(\mathrm{k})=\mathrm{d}_{j}^{(2)}(\mathrm{k})-\mathrm{X}^{(1) \mathrm{T}}(\mathrm{k}) \mathrm{W}_{j}^{(2)}(\mathrm{k}) \\
& \text { Weight of hidden layer : } W_{j}^{(1)}(\mathrm{k})=\mathrm{W}_{j}^{(1)}(\mathrm{k}-1)+\mathrm{g}^{(1)}(\mathrm{k})\left[\mathrm{d}_{j}^{(1)}(\mathrm{k})-\mathrm{X}^{T}(\mathrm{k}) \mathrm{W}_{j}^{(1)}(\mathrm{k}-1)\right]
\end{aligned}
$$

\section{Conclusion}

The method of fuzzy Neural Network embedding into a Intelligent, low-carbon urban lighting controlling system is :first using BP neural network algorithm to model and train light environmental detection data ,Taking Mamdani method in Matlab to make modeling analysis,conducting fuzzy inference and system configurations to achieve switching control for different light environment scene,To give priority to,use a distributed modular control, and complementary a centralized control as a bus control mode, fuse data in the controlling center,making controlling decision, providing a convenient way of controlling,using real-time data sources, analyse and process to obtain characteristic data consistent with the controlled object, making system performance complete and reliable,The control system has a self-learning, self-judgment and forecasting functions,so as to meet the needs of the city, people, vehicles to different light environment scene, achieve a low-carbon intelligent city lighting systems.

\section{References}

[1] Yan Na, Bai Xiangfang. Design and research of a wireless intelligent low carbon urban lighting system.Jiyuan journal of College of Vocational and Technical, 2012, 11(02): 23-26(In Chinese)

[2] Chi Kaili. Calibration of wireless sensor network based on BP neural network: [Master Thesis]. Zhejiang: University of Electronic Science and technology of Hanzhou, 2012(In Chinese)

[3] Luo Bing, Zhang Jianmin, Gan Junying. Intelligent control technology. 北京: Tsinghua University press, 2011(In Chinese) 